\title{
Influence of frame-dragging on magnetic null points near rotating black hole
}

\author{
V Karas, O Kopáček and D Kunneriath \\ Astronomical Institute, Academy of Sciences, Boční II 1401, CZ-141 31 Prague, \\ Czech Republic
}

\begin{abstract}
Understanding the mechanisms of particle acceleration from the vicinity of black holes poses a challenge. Electromagnetic effects are thought to be a prime suspect, but details still need an explanation. To this end, we study a three-dimensional structure of oblique magnetic fields near a rotating black hole in vacuum. It has been proposed that such a set-up can lead to efficient acceleration when plasma is injected near a magnetic null point. We focus our attention especially on the magnetic field in the immediate neighborhood of the magnetic null point, which was previously shown to occur in the equatorial plane. By employing the Line-Integral-Convolution (LIC) method, we visualize the magnetic field lines and explore the electric lines rising out of the equatorial plane.

We show the magnetic field structure near the boundary of ergosphere, depending on the spin of the black hole. Electric field develops a non-vanishing component passing through the magnetic null point and ensuring efficient acceleration of charged particles from this particular location near horizon. We also examine the effect of translatory boost on the field lines. Similarly to the frame-dragging by rotation, the linear motion carries field lines along with the black hole. Position of the magnetic null point recedes from the black hole horizon as the spin parameter increases. For the extreme value of $a=1$ the null point can occur outside the ergosphere.

PACS numbers: 04.20.-q, 97.60.Lf, 98.62.Js
\end{abstract}

\section{Introduction}

Magnetosphere is a region surrounding a magnetized body, where magnetic effects dominate the motion of electrically charged particles. Astrophysical black holes are electrically neutral and do not support their own magnetic field, nevertheless, they can be endowed by a magnetosphere when embedded in an external magnetic field that is generated by distant currents. Unlike planetary magnetospheres, black holes bring new quality by influencing the structure of electromagnetic fields directly by their strong gravity (Begelman et al 1984; Punsly 2008).

Near a rotating black hole, shearing motions are very intense, and capable of bending and folding magnetic lines frozen into plasma. This process is known to

create conditions suitable for magnetic reconnection (Koide and Arai 2008). But here we conceive a different setting: a magnetically dominated region, where large-scale 
magnetic fields get distorted by purely gravitational effects caused by proximity of the black hole. As a result of such an interaction, a complicated structure arises of narrow magnetic layers and associated neutral points (Karas \& Kopáček 2009). Reconnection can then occur when some amount of plasma is brought in the region, e.g. by the vacuum breakdown and the resulting production of electron-positron pairs.

Recently, Lyutikov (2011) investigated the role of motion of a Schwarzschild black hole through an ordered magnetic field. The linear velocity component leads to conditions permitting the energy extraction by an induced electrical field in the direction parallel to the magnetic lines. The released energy is eventually emitted in the form of an electromagnetic signal. However, the motion of a magnetized non-rotating black hole does not seem to produce magnetic neutral points. The formation of such a magnetic topology is an important aspect in the context of magnetic reconnection, which typically takes place where topologically distinct regions approach each other and the magnetic field lines change their connectivity (e.g., Priest and Forbes 2000).

Various processes can lead to the magnetic topology exhibiting null points, in particular, these can emerge by complex motions of the plasma. In this respect the black hole rotation brings a new situation, as magnetic field lines become twisted in a highly curved spacetime of a rotating black hole, approximately where the ergosphere forms. This is caused by gravito-magnetic interaction of general relativity (Islam 1985). Here, we examine the resulting structure of the magnetic field, namely, the emergence of critical points in a local frame of a physical observer, resembling the occurrence of Xpoints, which were studied, e.g. recently in the context of special-relativistic reconnection (Kojima et al 2011).

In the previous paper (Karas \& Kopáček 2009), we considered a special case of a uniform magnetic field in perpendicular orientation with respect to the black hole spin axis, and we demonstrated that magnetic null points can indeed form near a rotating (Kerr) black hole. Here, we identify precise location of the null points and we plot their radial position as a function of spin. The null point emerges, typically, for $a \gtrsim 0.2$ and it moves gradually away from the horizon until it crosses the boundary of the ergosphere from an almost extremely rotating black hole. Furthermore, in this paper the embedded magnetic field is allowed a general orientation, i.e., it can be inclined in an arbitrary angle with respect to the rotation axis. Therefore, axial symmetry is broken between the gravitational and electromagnetic fields, and this has to result in a truly threedimensional structure of magnetic field lines along with gravito-magnetically induced electric field (see Rieger 2011, for a recent review). In fact it was not obvious whether the magnetic nulls exist in the oblique case or whether their occurrence is an artifact of special (perpendicular) orientation of the magnetic field. The adopted approach allows us to identify the gravitational effects operating in a magnetically dominated system, where a super-equipartition magnetic field governs the motion of plasma. We indeed find the null points also in a fully three-dimensional case of an inclined magnetic field. 


\section{Aligned and oblique magnetic fields near black hole horizon}

Astrophysical black holes do not support their own intrinsic magnetic field; this has to be generated by external currents and brought down to horizon by accretion. An active role of accretion on transporting large-scale magnetic fields toward horizon has been recognized (Bisnovatyi-Kogan and Lovelace 2007; Rothstein and Lovelace 2008). A black hole can also enter a pre-existing magnetic flux tube, and then one asks if the process of magnetic reconnection is influenced by strong gravitational field near horizon. And does the black hole rotation play a significant role in the scenario of this kind?

As we wish to discuss magnetic fields inclined with respect to the spin axis, and we also want to include the fast translatory motion, the following picture appears to be appropriate: Kerr black hole traversing a "magnetic filament", described as an extended (largely one-dimensional) flux tube. Such an idea can be motivated observationally, by highly ordered and elongated arcs (of about $100 \mu \mathrm{G}$ ) that are seen in Galactic Center, within a few parsecs from Sagittarius A* compact radio source (Sgr A*; Yusef-Zadeh et al 1984). They are thought to represent large-scale magnetic flux tubes

that are illuminated by synchrotron emission from relativistic electrons (LaRosa et al 2004; Morris 2006; Ferrière 2010).

Given a limited resolution that can be achieved with current imaging techniques, the magnetic filaments cannot be traced down to the characteristic size of the black hole Therefore, the actual mapping of the magnetic structures near black holes is not directly possible (this may change with new interferometrical techniques in near future). The role of magnetic structures in accelerating the particles is suggestive, especially because they could help us to understand the origin of particle acceleration and the resulting signatures in the electromagnetic signal.

Naturally, in astrophysically realistic solutions the role of non-ideal plasma will need be included. Currently, neither of the frequently discussed limits (i.e., vacuum vs. force-free approximations) are able to account for both the plasma currents as well as the accelerating electric fields. This task will require resistive MHD, which is, however, beyond the scope of this brief paper.

\subsection{The effect of black hole rotation and translatory motion}

Starting from King et al (1975) and Bičák \& Dvořák (1976), the organised electromagnetic test fields surrounding black holes have been discussed and their astrophysical consequences considered in various papers. The case of oblique geometry, however, has been explored only partially (Bičák and Janiš 1980). This is caused especially by the fact that the off-equatorial fields are lacking any symmetry, and so are more difficult to

$\ddagger$ Gravitational radius $r_{\mathrm{g}}=G M / c^{2} \approx 1.48 \times 10^{13} M_{8} \mathrm{~cm}$, where the central black hole mass is expressed in terms of $M_{8} \equiv M /\left(10^{8} M_{\odot}\right)$. The velocity of the Keplerian orbital motion of a particle is then $v_{\mathrm{K}} \approx 2.1 \times 10^{10}\left(r / r_{\mathrm{g}}\right)^{-1 / 2} \mathrm{~cm} \mathrm{~s}^{-1}$. The corresponding orbital period is $T_{\mathrm{K}} \approx 3.1 \times 10^{3}\left(r / r_{\mathrm{g}}\right)^{3 / 2} M_{8} \mathrm{~s}$. Hereafter, we use a dimensionless form of geometrized units, where all quantities are scaled with the black hole mass; $M$ does not appear in the equations explicitly. 

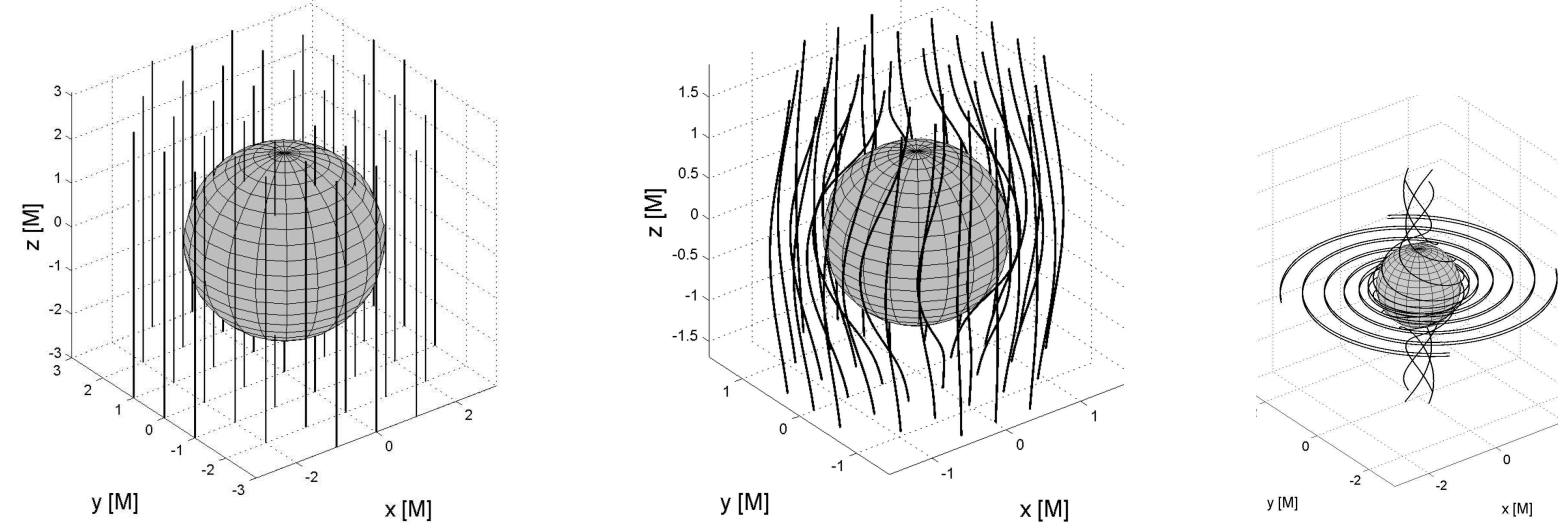

Figure 1. Asymptotically uniform magnetic field aligned with rotation axis of the black hole in vacuum. Tetrad components are plotted with respect to frame of freefalling observers in stereometric projection. Left: magnetic lines near a non-rotating black hole appear to be perfectly homogeneous (in these suitably defined coordinates). Middle: magnetic field aligned with the rotation axis of an maximally rotating $(a=1)$ Kerr black hole, when they are expelled out of the horizon. Right: electric lines corresponding to the magnetic field shown in the middle panel. A toroidal component is induced by the gravito-magnetic action of the rotating black hole (see Kopáček 2011, for more details).

visualise. Also, qualitatively new effects on the field structure were not expected.

Nevertheless, in Karas \& Kopáček (2009) we were able to demonstrate that the asymptotically perpendicular field lines develop a magnetic neutral point in the equatorial plane. This is interesting because such structures of the magnetic field are relevant for processes of electromagnetic acceleration. The magnetic null point emerges in a local physical frame, and could trigger reconnection. However, the asymptotically perpendicular field is a rather exceptional case. Therefore, here we investigate nearhorizon magnetic structures in more detail, also for a general orientation of the magnetic field.

The main objective of this discussion is to track the location of magnetic null points and to explore a complex three-dimensional configuration inside the ergosphere. We consider the form of magnetic lines together with the induced electric lines for different values of the model parameters: the inclination angle of the asymptotic magnetic field $\theta_{\mathrm{o}}\left(=\arctan \left(B_{\perp} / B_{\|}\right)\right.$, the black hole spin $a\left(a^{2} \leq M\right)$, and the boost velocity $\beta$ $\left(\beta^{2} \equiv v_{x}^{2}+v_{y}^{2}+v_{z}^{2}<1\right)$. We observe the layers of alternating magnetic orientation to occur also in the general case, i.e., when the black hole rotates and moves with respect to an oblique magnetic field. However, the three-dimensional structure of the field lines is very complicated as they become highly entangled around the null point.

We specify the gravitational field by Kerr metric (Misner et al 1973). Our starting form of the electromagnetic field is a stationary solution of Maxwell's test-field electro- 

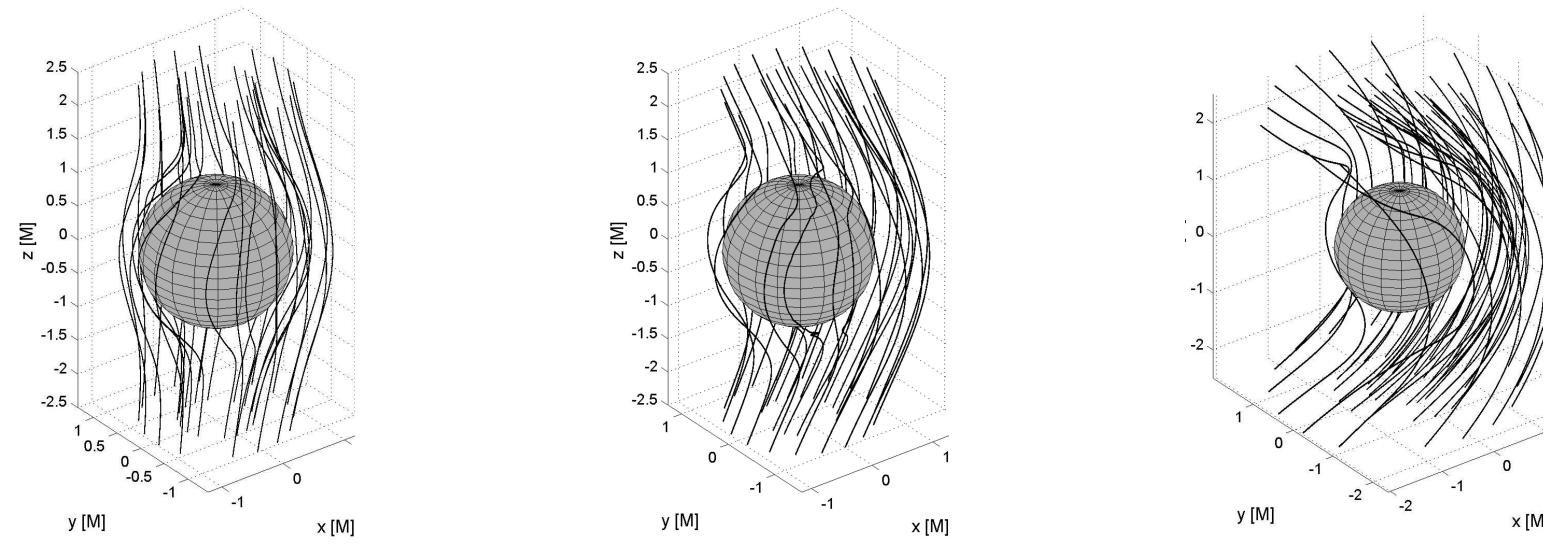

Figure 2. The effect of black hole motion on magnetic lines from the previous figure. The case of extreme spin, $a=1$, with a gradually growing velocity of the translatory boost along $x$-direction: $v_{x}=0.1$ (left), $v_{x}=0.3$ (middle), and $v_{x}=0.7$ (right).

vacuum equations in the given curved spacetime $\$$ The electromagnetic four-potential $\boldsymbol{A}$ can be then written as superposition of two contributions: $\boldsymbol{A}=B_{\|} \boldsymbol{a}_{\|}+B_{\perp} \boldsymbol{a}_{\perp}$, where $B_{\|}$and $B_{\perp}$ define the magnitudes of the two parts.

The aligned field has two non-vanishing components of the normalized electromagnetic four-potential,

$$
\begin{aligned}
& a_{t \|}=B_{\|} a\left[r \Sigma^{-1}\left(1+\mu^{2}\right)-1\right], \\
& a_{\phi \|}=B_{\|}\left[\frac{1}{2}\left(r^{2}+a^{2}\right)-a^{2} r \Sigma^{-1}\left(1+\mu^{2}\right)\right] \sigma^{2},
\end{aligned}
$$

where we use Boyer-Lindquist $t, r, \theta$, and $\phi$ dimension-less spheroidal coordinates $(\mu=\cos \theta, \sigma=\sin \theta)$. Eqs. (1) -(2) represent an asymptotically homogeneous magnetic field (Wald 1974).

On the other hand, the perpendicular to axis component of the field is given by (Bičák and Janišs 1980)

$$
\begin{aligned}
& a_{t \perp}=B_{\perp} a \Sigma^{-1} \Psi \sigma \mu, \\
& a_{r \perp}=-B_{\perp}(r-1) \sigma \mu \sin \psi, \\
& a_{\theta \perp}=-B_{\perp}\left[\left(r \sigma^{2}+\mu^{2}\right) a \cos \psi+\left(r^{2} \mu^{2}+\left(a^{2}-r\right)\left(\mu^{2}-\sigma^{2}\right)\right) \sin \psi\right], \\
& a_{\phi \perp}=-B_{\perp}\left[\Delta \cos \psi+\left(r^{2}+a^{2}\right) \Sigma^{-1} \Psi\right] \sigma \mu,
\end{aligned}
$$

with $\Sigma(r, \mu)$ and $\Delta(r)$ being the Kerr metric functions, $\psi \equiv \phi+a \delta^{-1} \ln \left[\left(r-r_{+}\right) /\left(r-r_{-}\right)\right]$, $\Psi=r \cos \psi-a \sin \psi, \delta=r_{+}-r_{-}$, and $r_{ \pm}=1 \pm \sqrt{1-a^{2}}$. Knowing the complete set of four-potential components the magnetic field structure is fully determined: $F_{\mu \nu} \equiv A_{[\mu, \nu]}$.

Equations (3) -(6) describe the field lacking the axial symmetry. Such a situation cannot be strictly stationary, however, the alignment time-scale is very long, and so we

$\S$ These are astrophysically acceptable approximations which reflect the fact that black holes can only acquire a negligibly small electric charge, while cosmic electromagnetic fields are not strong enough to influence the spacetime metric significantly. 

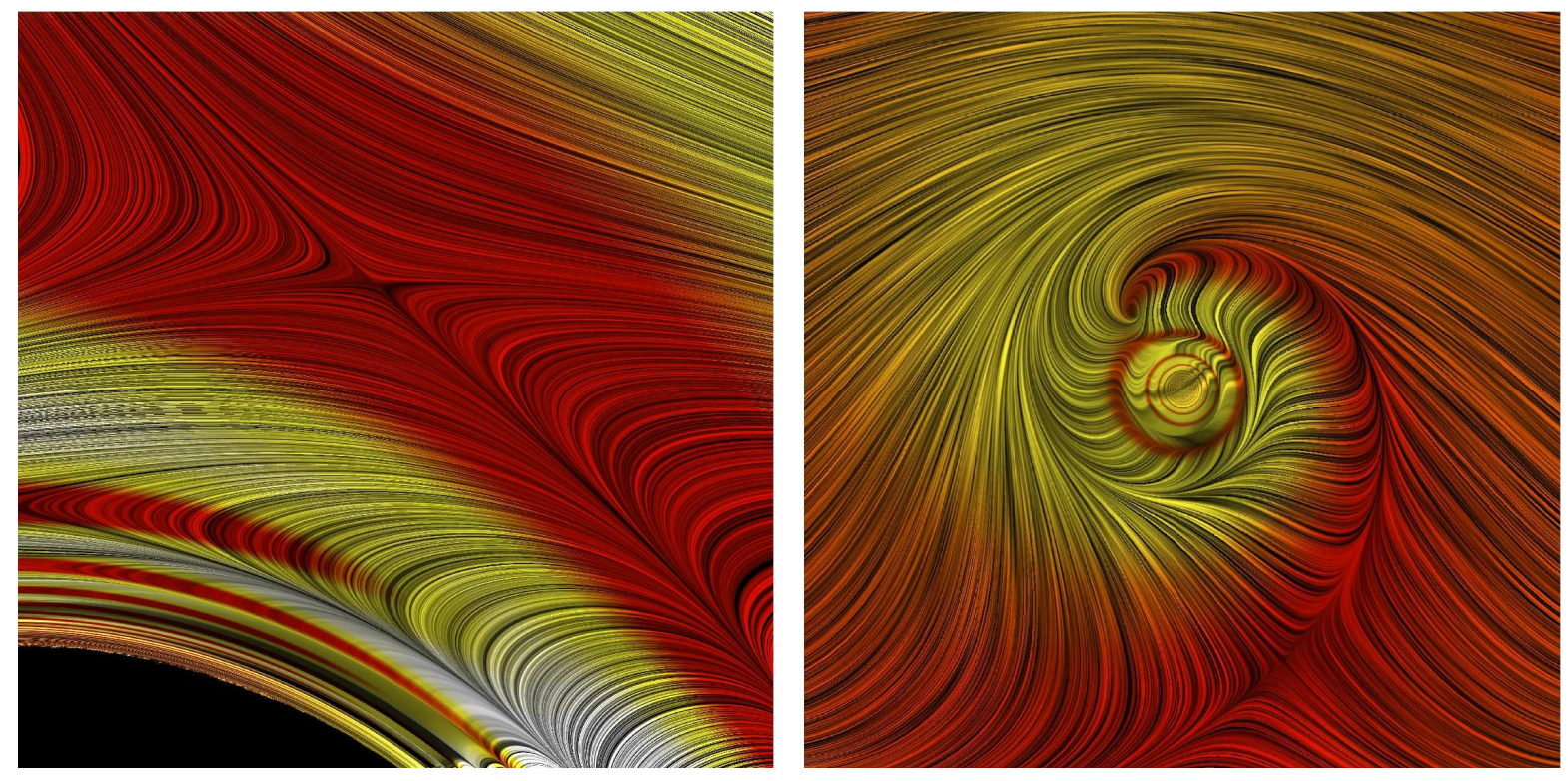

Figure 3. Perpendicular magnetic field in the equatorial plane $(x, y) \equiv$ $(r \cos \phi, r \sin \phi)$, i.e., viewed along the rotation axis of an extreme $(a=1)$ black hole. While the field is steady and uniform far from the black hole, its structure becomes highly entangled near horizon. Left panel: the neighborhood of the magnetic null point, which reveals itself clearly in the upper part of the plot. The black circular section in the bottom left corner denotes the horizon, $r=r_{+}(a)$. Right panel: the layered structure of the magnetic field close above the horizon is revealed by using the radial coordinate $\xi(r) \leq 0.6$ (i.e., $r_{+}<r \leq 2.5 G M / c^{2} ; r=r_{+}$collapses to the center of the plot). The magnetic null is now exhibited in the lower part of the plot. The colour scale indicates the magnetic intensity (in arbitrary units), ranging from vanishingly small values (red) across the moderate (yellow) to the maximum (white).

can neglect the associated energy losses here (Kim et al 2003). The field line structure depends also on the motion of observers determining the field components. In order to construct and discuss our plots, we employ a free-falling physical frame, evaluate the electromagnetic tensor with respect to the local frame, and use these components to draw the field lines.

In absence of perpendicular component $\left(B_{\perp}=0\right)$, the field is relatively uncomplicated (see figure 11). Although the frame-dragging acts also on the aligned field lines, their shape can be integrated to find the surfaces of constant magnetic flux in a closed (analytical) form. Previously, the aligned fields were explored especially in context of magnetic field expulsion from a maximally rotating black hole (and how this is changed when a conducting medium surrounds the black hole; cf. Komissarov and McKinney 2007). In our notation, the example in Fig. 1 refers to zero velocity of the translatory boost, i.e. $\beta=0$. Once we include a nonzero boost velocity, the structure of the aligned field becomes more complicated (figure 2). This is mainly due to an interplay between the boost of the black hole and its rotation acting jointly on the (originally) aligned field. As $\beta$ increases, the magnetic lines are progressively puffed out of horizon and wound around it (see Kopáček 2011, for more examples and 

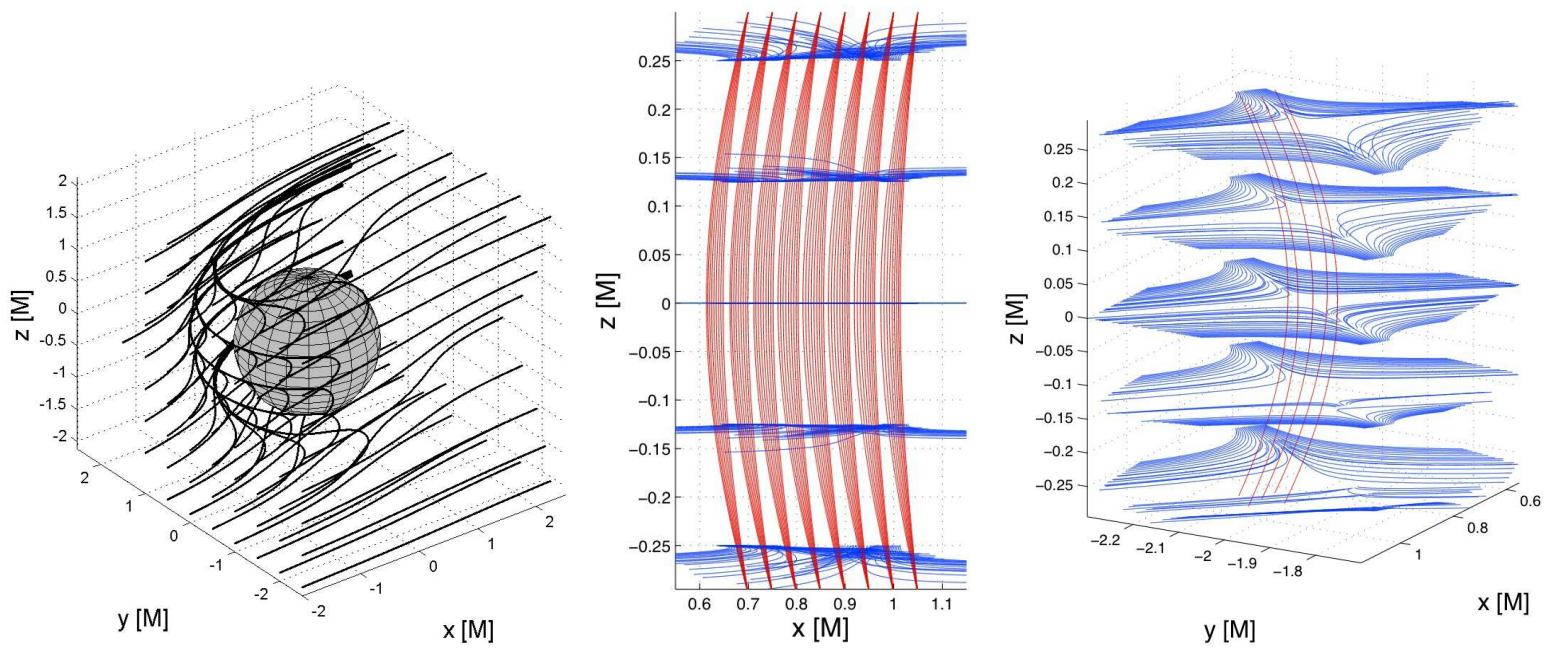

Figure 4. Left panel: magnetic field asymptotically perpendicular to the rotation axis $(z)$ of an $a=1$ black hole, centered on the origin. Clearly seen is the effect of dragging by rotation of the black hole. Middle: side-view projection of the equatorial $(z=0)$ plane in which magnetic field lines (blue) reside, while the induced electric lines (red) are rising out of the plane. Outside the equatorial plane, also the magnetic field acquires some non-zero vertical component allowing the particle acceleration. Right: a three-dimensional detail around the equatorial plane reveals a magnetic null point. Again, the electric lines are shown to pass through the null point in the vertical direction.

details).

\subsection{Neutral points of the magnetic field}

Let us now explore the case of magnetic field with a non-vanishing component inclined with respect to rotation axis $\left(B_{\perp} \neq 0\right)$. In fact, Karas \& Kopáček (2009) explored a strictly perpendicular case. Confining the magnetic lines in the equatorial plane $\theta=\pi / 2$, the nested structure of magnetic layers emerges. These are essential for the magnetic reconnection.

Near-horizon structure of magnetic lines is visualized in figure 3 by using the LineIntegral-Convolution (LIC) method in Matlab. This technique allows us to identify clearly the location of neutral points. It turns out to be particularly useful here with the general orientation of the asymptotic field direction, as the global solution for the field lines is too cumbersome. Further, by introducing $\xi(r ; a) \equiv 1-r_{+}(a) / r$ as a new radial coordinate, the horizon surface $r=r_{+}$collapses into the center and the layered structure of magnetic sheets is seen in more detail.

A fully three-dimensional structure of magnetic lines develops outside the equatorial plane. In figure 4 we observe a superposition of two essential effects. Firstly, the X-type structure of the magnetic null point persists also outside the equatorial plane. Secondly, however, the magnetic lines acquire a growing vertical component $B_{z}\left(=B_{\|}\right)$, whereas the electric field passes through the magnetic null point and crosses the equatorial 


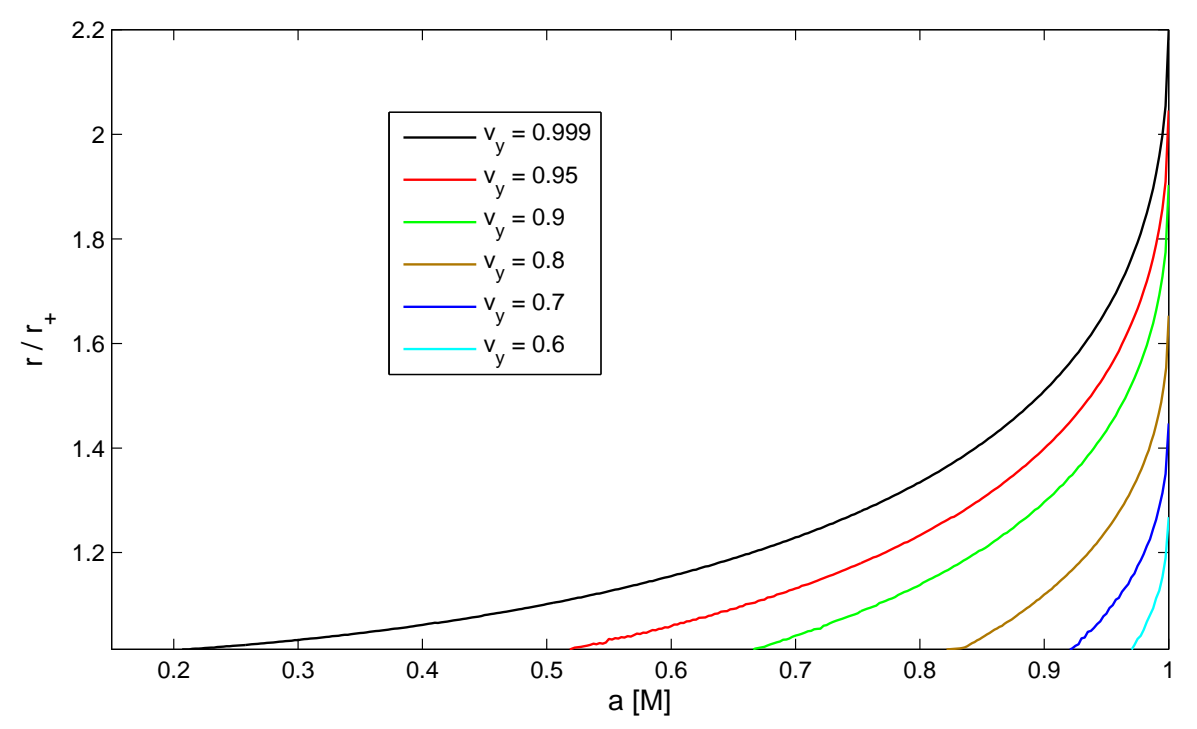

Figure 5. Position in the equatorial plane of the magnetic null point corresponding to a magnetic field asymptotically perpendicular with respect to rotation axis. Fast translatory motion and fast rotation are both important for the emergence of null points. Several curves are shown as a function of the black hole spin $a$ for different velocity $v_{y}$ (i.e., in the direction along $y$-axis), which is set in the direction perpendicular with respect to the rotation axis, as well as perpendicularly to the asymptotic uniform magnetic field.

plane vertically. Such a structure suggests that particles can be accelerated by the non-vanishing electric field, and they can stream away from the neutral point.

It is interesting to investigate the location of magnetic null points as a function of the black hole spin $a$ and velocity $v$ of the translatory motion. This question is especially relevant in the context of the electromagnetic acceleration mechanisms of particles in the vicinity of rapidly rotating black holes (position of the magnetic null point recedes from the horizon as the spin $a$ increases). We would like to identify the site of magnetic reconnection and ask whether it is located outside the ergosphere or inside it, depending on the black hole spin. The result is shown in figure 5, where we plot the radial distance of the magnetic null points as a function of spin for different values of the linear (boost) velocity $v_{y}>0\left(v_{x}=v_{z}=0\right)$. Orientation of the velocity is set perpendicular to the asymptotic field, $B_{x}>0, B_{z}=0$. This special configuration of a magnetic field (mutually orthogonal with respect to the drift velocity and the rotation axis) provides suitable conditions for the emergence of the null points. In the case of extreme spin we observe the occurrence of null points for velocity as low as $v_{y} \approx 0.45$. On the other hand, rapid motion $v_{y}=0.999$ allows the null point formation also for a rather low spin about $a \approx 0.2$. Both $a$ and $v$ parameters are essential to ensure the existence of null points, and they both help to drag the point farther out from the horizon. A combination of extreme spin $a \rightarrow M$ and fast motion $v_{y}=0.999$ brings the magnetic null to $r=2.203$ $\left(G M / c^{2}\right)$. Increasing the velocity beyond this value does not move the neutral point any farther. On the other hand, for small values of spin and/or small velocity the null 

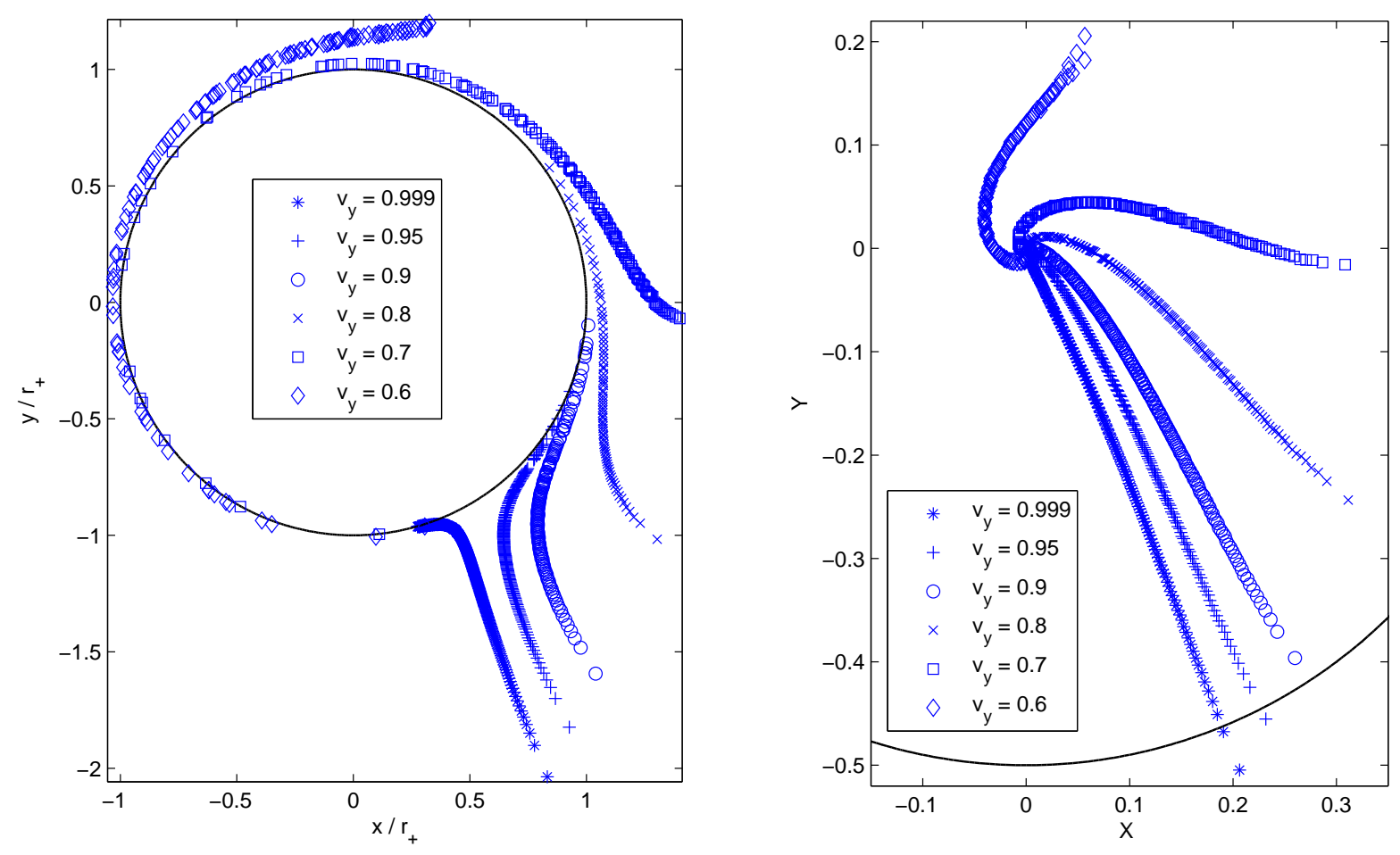

Figure 6. Position of the magnetic null points which emerge in the equatorial plane as the spin and the drift velocity are gradually increased. Different markers indicate the magnitude of translation velocity as specified in the inset (only $v_{y}$ component of the velocity in the equatorial plane is assumed to be non-zero). Left panel: Given the velocity, null points form separate traces of a gradually increasing spin $a$ : outer endpoints correspond to the extreme spin $a=1$; the spin decreases monotonically along each track as one approaches the horizon (denoted by the black circle). Right panel: The same as on the left side, but now plotted with respect to the rescaled polar radial coordinate $\xi$. This helps us to resolve more clearly the narrow region just above the horizon (in the origin of the graph). The outer circle indicates the equatorial radius of the ergosphere of the extreme spin black hole.

point does not show up at all.

Figure [6]completes information about the null point location in the equatorial plane. Magnetic field is again specified to be asymptotically perpendicular to the rotation axis $\left(B_{x}>0, B_{z}=0\right)$ and the black hole moves transversely with respect to both the magnetic field and the rotation axis $\left(v_{y}>0, v_{x}=v_{z}=0\right)$. The view of the equatorial plane in polar coordinates allows us to demonstrate how the magnetic null points move gradually away from the horizon as the spin increases, while they are dragged around the hole by its frame-dragging effect. For high velocity and large spin the null points emerge just outside the ergosphere boundary, whereas for lower values they are generally confined within the ergosphere. We also observe that the distance of null points in oblique configurations of the system is generally smaller compared to the perpendicular case shown here, so we conclude that fig. 6 shows the farthest location to which the magnetic null point can recede from the black hole horizon.

To conclude the discussion of the position of magnetic null points, in figure 7 we 

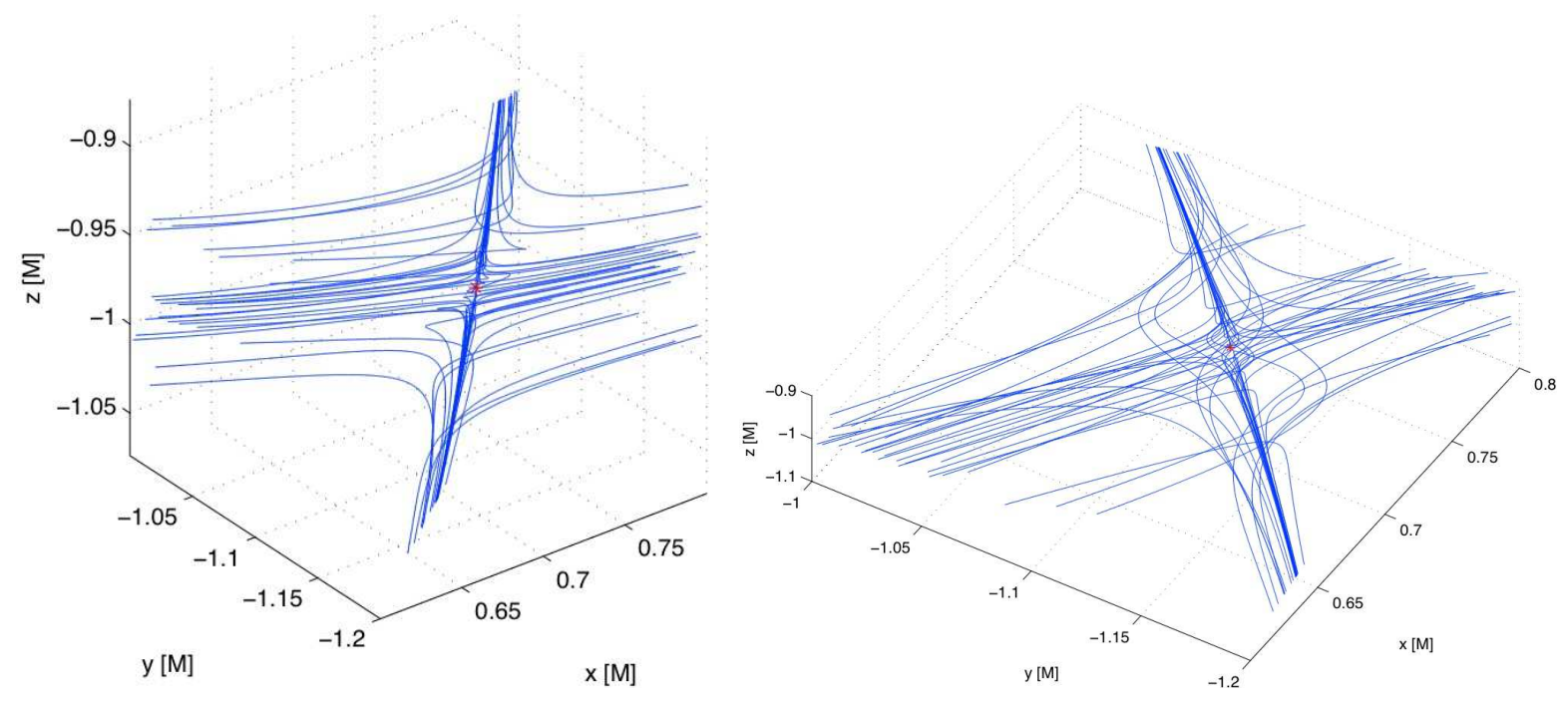

Figure 7. Structure of the oblique magnetic field near the null point (depicted by the red marker). The null point occurs near above the horizon. Here we consider the extremal black hole with a rapid drift velocity $\left(v_{x}=v_{y}=0.7, v_{z}=0\right)$ and the background of an inclined magnetic field $\left(B_{x}>0, B_{x}=B_{z}\right)$. Two different views of the same system are shown.

explore a general case when the spin of the black hole, the direction of its translatory motion, and the asymptotic magnetic field point all in different arbitrary directions. This case corresponds to the oblique system which lacks any special symmetry. Despite that, it is interesting to note that the magnetic null points again exist near the horizon (albeit out of the equatorial plane). Also in the oblique case, by raising the spin $a$ we locate the magnetic null points getting gradually farther away from the horizon (see figure (8). The null points can be traced in analogy with those discussed previously in the equatorial case (fig. 6), however, now the shape of the resulting tracks is more distorted and the null points positions are more difficult to find. Because locating the null points of the oblique field does not seem to be possible directly from the analytical form of the field components, we have searched the relevant region numerically.

Finally, it may be worth reminding the reader that the exact location of magnetic nulls depends on the choice of frame with respect to which we formulate the problem. We considered a well-motivated physical frame and we checked that the null points occur also, e.g., in the frame of freely orbiting (Keplerian) observers, so the effect is relevant for the behaviour of accreted matter.

\section{Discussion and Conclusions}

Rotation is an interesting attribute of cosmic black holes (Reynolds \& Nowak 2003). In principle, black holes can be spun up close to extreme $a=1$ (Sadowski et al 2011). In stellar-mass black holes the spin is thought to be chiefly natal (McClintock et al 2006), whereas supermassive black holes in galactic cores can adjust their angular momentum 


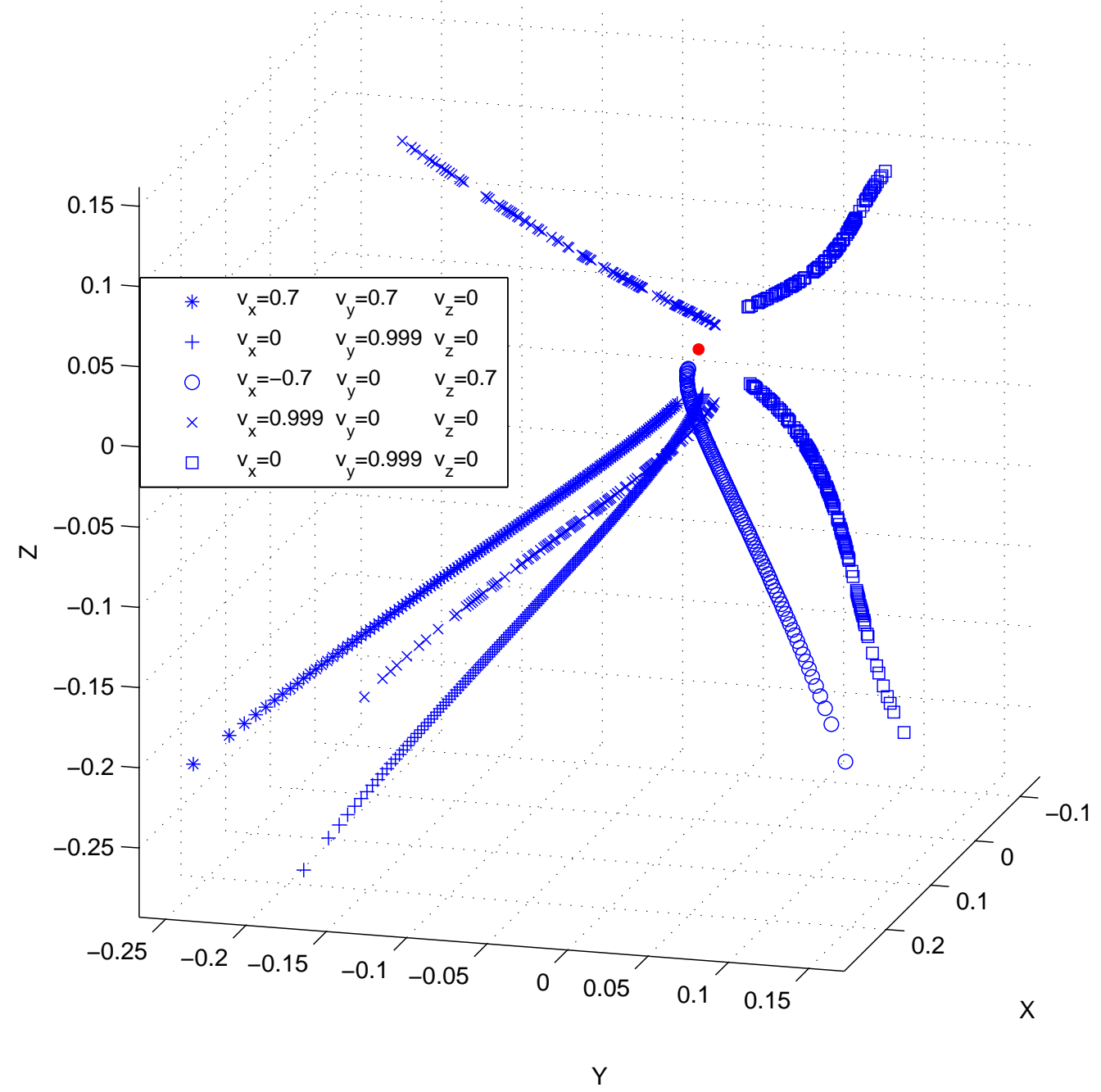

Figure 8. The position of null points in a general case, plotted with respect to the mutual orientation of the black hole's drift velocity and the asymptotic magnetic field. The velocity components are indicated in the inset; from top to the bottom, the first three cases are for the oblique field $\left(B_{x}>0, B_{x}=B_{z}\right)$, while the remaining two examples show the aligned field $\left(B_{z}>0, B_{x}=0\right)$ for comparison. In the latter case the tracks of null points form pairs that are positioned symmetrically with respect to the equatorial $(z=0)$ plane. The outer end of each track corresponds to the extreme spin, $a=1$. The spin decreases monotonically along each series of points towards the horizon. The rescaled radial coordinate has been employed, so the horizon corresponds to a single point in the origin of coordinates (depicted by the red dot).

by accretion, and the outcome of evolution depends largely on the dominant mode of accretion, during their entire life-time (Fanidakis et al 2011). In both cases, the spin is an important characteristic, potentially allowing the efficient acceleration of matter. We showed that it can be also relevant directly for the onset of magnetic reconnection.

We discussed the structure of electromagnetic test-fields and the layered pattern of current sheets that can be induced by the gravito-magnetic action. Neutral points of the magnetic field suggest that magnetic reconnection can occur. In the case of 
extreme rotation, $a=1$, the magnetic null points can occur just outside the ergosphere. We found that the null points exist also in a general (oblique) case which lacks any special symmetry between the direction of the magnetic field, velocity of the linear boost of the black hole, and the orientation of the spin axis. The proposed scenario can be astrophysically relevant in circumstances when the black hole rotates and moves simultaneously across a magnetic flux tube. The origin of the magnetic flux tube is thought to be in currents flowing in the medium far from the black hole. We considered the limit of a magnetically dominated system in which the organised magnetic field dictates the motion to plasma; the opposite limit of a black hole moving through a forcefree plasma has been investigated by other authors (recently, Palenzuela et al 2010).

In this paper we considered an idealised situation, starting from an electrovacuum solution, assuming fast motion and rotation of the black hole, and embedding it in an asymptotically uniform magnetic field. Future simulations should clarify, whether the astrophysically realistic effects of the moving black hole on the surrounding electromagnetic structure in its immediate neighborhood will be similar to those envisaged here. Despite the field-line structure in this paper being induced purely by the action of frame-dragging, the exact choice of the projection tetrad is not very essential for the existence of magnetic layers. The choice of the physical frame does affect the presence and the exact location of the magnetic neutral point, and the associated electric field which accelerates charged particles away from the neutral point. Although the exact location of the neutral point varies with the model parameters, it always occurs very close to the black hole, where the frame-dragging is efficient. Therefore, the point of particle acceleration has to be close to horizon as well.

We note that all essential ingredients needed for the aforementioned mechanism to operate are met in standard circumstances that are believed to occur in various cosmic objects. Namely, large scale magnetic fields are frequently observed. Furthermore, binary black holes are expected to exist in some nuclei of galaxies, where they are gravitational bound to each other and perform the orbital motion, while gradually inspiralling inwards; shortly before the merger, the motion speeds up significantly. Analytical models for this phase are not possible - in general the spacetime structure has to be solved numerically, nevertheless, the magnetic structure of the organised field may arise quite generically in the close vicinity of such fast-moving black holes that are embedded within an external magnetic field (Mösta et al 2010). Magnetic filaments exist in our galactic center and they suggest that the approximation of an asymptotically uniform organised magnetic field is worth investigating in this context.

\section{Acknowledgments}

We thank the Czech Science Foundation grant (No. 205/09/H033) and the Czech-US Cooperation Program (ME09036) for a continued support. We thank our referees for constructive comments. 


\section{References}

Begelman M C, Blandford R D and Rees M 1984 Rev Mod Phys 56255

Bičák J and Dvořák L 1976 GRG 7959

Bičák J and Janiš V 1980 MNRAS 212899

Bisnovatyi-Kogan G S and Lovelace R V E 2007 ApJL 667 L167

Fanidakis N, Baugh C M, Benson A J et al 2011 MNRAS 41053

Ferrière K 2010 AN 33127

Islam J N 1985 Rotating Fluids in General Relativity (Cambridge: C.U.P.)

Karas V and Kopáček O 2009 CQG 26025004

Kim H, Lee H K, Lee C H 2003 JCAP 9001

King A R, Lasota J P and Kundt W 1975 Phys Rev D 123037

Koide S and Arai K 2008 ApJ 6821124

Kojima Y, Oogi J and Kato Y E 2011 A $\& A 531$ A47

Komissarov S S and McKinney J C 2007 MNRAS 377 L49

Kopáček O 2011 Thesis (Charles University, Prague), arXiv:1110.4553

LaRosa T N, Nord M E, Lazia T J W and Kassim N E 2004 ApJ 607302

Lyutikov M 2011 Phys Rev D 83064001

McClintock J E, Shafee R, Narayan R et al 2006 ApJ 652518

Misner C W, Thorne K S and Wheeler J A 1973 Gravitation (New York: Freeman)

Morris M 2006 J Phys Conf Ser 541

Mösta P, Palenzuela C, Rezzolla L et al (2010) Phys Rev D 81064017

Palenzuela C, Garrett T, Lehner L and Liebling S L 2010 Phys Rev D 82044045

Priest E and Forbes T 2000 Magnetic Reconnection (Cambridge: C.U.P.)

Punsly B 2008 Black Hole Gravitohydromagnetics (New York: Springer)

Reynolds C S and Nowak M A 2003 Physics Reports 377389

Rieger F M 2011 Int J Mod Phys D 201547

Rothstein D M and Lovelace R V E 2008 ApJ 6771221

Sadowski A, Bursa M, Abramowicz M et al 2011 A $\& A 532$ A41

Wald R M 1974 Phys Rev D 101680

Yusef-Zadeh F, Morris M and Chance D 1984 Nature 310557 